\title{
Migración y transculturación del tópico del león reverente y agradecido en la literatura fundacional rioplatense de Ruy Díaz de Guzmán*
}

\section{Migration and Transculturation of the Reverent and Grateful Lion in Ruy Díaz de Guzmán's Founding Literature of the River Plate Region}

\section{ELISABETH KRUSE}

Romanisches Institut

Ludwig-Maximilians-Universität München

Schellingstr. 3, Múnich, Baviera 80799. Alemania

e.kruse@Imu.de

Orcid ID 0000-0003-4629-1336

Resumen: En el contexto de la migración y globalización de la cultura hispánica y, a través de esta, de los mitos y tópicos más persistentes de la civilización europea, encontramos la obra del mestizo asunceno Ruy Díaz de Guzmán Irala. La inserción del tópico del león reverente y agradecido sufrirá un proceso de transculturación al nuevo lugar del hispanismo, siendo adaptado a las circunstancias históricas, en las que reina el enfrentamiento con la alteridad, representada por los pueblos originarios. Como fuentes más directas, pondremos el texto hispanoamericano en diálogo con El libro de los ejemplos de Clemente Sánchez de Vercial y Corónica del Çid Ruy Díaz, entre otros. La recontextualización de este tópico en tierras rioplatenses fungirá como vehículo de exaltación de la epopeya española en estas regiones y la reinserción en el nuevo espacio geográfico-social posibilitará la plasmación de una visión dicotómica (civilización y barbarie) impregnada de una continua tensión.

Palabras clave: Migración. Transculturación. Tópicos. Exempla. Identidad.
RECIBIDO: 11 DE ENERO DE 2018 ACEPTADO: 19 DE SEPTIEMBRE DE 2018
Abstract: In the context of migration and globalization of the Hispanic culture, and through it, of the most persistent myths and topics of European civilization, we find the work of the mestizo asunceno Ruy Díaz de Guzmán Irala. The inclusion of the topic of the reverent and grateful lion will undergo a process of transculturation in the new Hispanic space and will be adapted to the historical circumstances, in which the confrontation with the other -represented by the indigenous people- reigns. We will compare the Hispanic-American text with El libro de los ejemplos by Clemente Sánchez de Vercial and Corónica del Çid Ruy Díaz, among others. The recontextualization of this topic in the Argentine pampas will serve as a means to exalt the Spanish feat in the Río de la Plata area and the reintegration in the new geographic and social space will enable the depiction of a dichotomic vision (civilization and barbarism) imbued with a continuous tension.

Keywords: Migration. Transculturation. Topics. Exempla. Identity.

\footnotetext{
* Trabajo realizado en el marco de una estancia de investigación sobre literatura argentina de los siglos XVII y XIX, financiada por la Cátedra de Altos Estudios del Español de la Universidad de Salamanca.
} 
a obra del primer cronista mestizo del Río de la Plata, el asunceno Ruy Díaz de Guzmán Irala, autor de los llamados Anales del descubrimiento, población y conquista de las provincias del Río de la Plata, fechado por él en 1612 y conocido a partir del siglo XVIII como La Argentina manuscrita, se enmarca en el proceso de migración y globalización de la cultura hispánica y, a través de esta, de los mitos y tópicos más persistentes de la civilización europea.

En vistas a abordar este fenómeno, nos proponemos analizar la inserción en su obra del tópico del león reverente y agradecido, concretamente en el famoso episodio de "La Maldonada", cuyo argumento más adelante recordaremos. Este tópico posee fuerte resonancia veterotestamentaria y grecolatina, además de asidua presencia en la literatura española medieval y del Siglo de Oro. Intentaremos en este espacio, presentar dos tesis acerca de este episodio: en primer lugar, a pesar del amplio periplo migratorio del tópico, determinar cuál pudo haber sido la fuente literaria de Díaz de Guzmán, la cual creemos que es, de manera directa, El libro de los exenplos de Clemente Sánchez de Vercial, donde, a su vez, de manera indirecta, confluyen la tradición grecolatina, por una parte, y la veterotestamentaria por otra, y que ejemplificaremos con el Libro de Daniel y la Corónica del Çid Ruy Díaz. En segundo lugar, intentaremos señalar las importantes funciones que cumple la transculturación de estos tópicos en América, nuevo lugar de la hispanidad.

La transculturalidad consiste en recurrir a modelos que no son generados en el propio contexto cultural, es decir en la cultura local o de base, sino que provienen de culturas externas y corresponden a otra identidad o lengua. (Toro 16)

Antes de entrar en el análisis de estos tópicos en la obra de Guzmán, hemos de explicar a continuación brevemente sus antecedentes literarios y su contenido. La plasmación literaria más famosa del tópico del león agradecido es la de la conocida historia del esclavo Androcles, narrada por Aulo Gelio (126-?) en sus Noches áticas. El esclavo, escapando de los malos tratos de su amo en Roma, huye al desierto en Libia, donde el calor lo obliga a refugiarse en una cueva, morada de un león. Por fortuna, este llevaba clavada una espina en su pata, cuya dolorosa herida le sana el esclavo. En agradecimiento, el león lo alimentará durante tres años de pacífica convivencia. No obstante, harto de esta vida de fieras, Androcles decide abandonar la cueva del león, pero es atrapado y condenado a morir en el Circo Máximo en las fauces de los leones, por haber escapado de su amo. Sin embargo, otra vez quiso la fortuna que fuera 
este mismo león la fiera destinada a devorarlo. Ante el pánico de Androcles y frente a todos, el león no solo no lo devora, sino que, tras reconocerlo, lo reverencia. Este hecho tan asombroso cautiva la buena voluntad del público, quien solicita ardorosamente la inmediata libertad del esclavo y del león. Este relato (a menudo erróneamente atribuido a Esopo y cuyo origen remoto no puede precisarse con exactitud pero que posiblemente pertenezca a la colección de cuentos de la India del Rig Veda), aparece documentado por primera vez en la literatura latina en el siglo II d.C.

Presentamos a continuación un esquema con las principales fuentes antiguas y medievales del tópico del león agradecido y reverente. No ha de sorprendernos la variedad de fuentes de las que pudo haber bebido Ruy Díaz antes de componer su relato ya que en la América colonial reinaba una verdadera afición por libros de muy diverso origen:

La voracidad popular por leer novelas de caballerías hacía necesaria una creciente producción, y los temas originales se retocaban o se mezclaban con otros, con uno que otro nuevo detalle fantástico o sentimental. No bastaban las fuentes de inspiración españolas y francesas; también se echó mano de las literaturas italiana, griega, romana, oriental. (Irving103)

\begin{tabular}{|c|c|c|}
\hline AUTOR & OBRA & TÓPICO \\
\hline Séneca (4 a.C.-65 d.C.) & De beneficiis, L. II, 19 & $\begin{array}{l}\text { León defiende a su domador } \\
\text { del ataque de las fieras }\end{array}$ \\
\hline Aulo Gelio (c. 126-¿?) & Noches áticas & Historia de Androcles y el león \\
\hline Claudio Eliano (170-249) & De natura animalium, L. VII, 48 & $\begin{array}{l}\text { Historia similar a la de Androcles } \\
\text { y el león (con variantes) }\end{array}$ \\
\hline $\begin{array}{l}\text { Chrétien de Troyes } \\
\text { (c. 1150-1183) }\end{array}$ & Yvain, el caballero del león & León agradecido \\
\hline \multirow[t]{2}{*}{$\begin{array}{l}\text { Clemente Sánchez de Vercial } \\
\text { (1370-1426) }\end{array}$} & $\begin{array}{l}\text { Libro de los Exemplos por A.B.C., } \\
\text { exemplum } 186\end{array}$ & $\begin{array}{l}\text { Historia de Androcles y el león } \\
\text { (con variantes) }\end{array}$ \\
\hline & Exemplum 185 & Loba parturienta agradecida \\
\hline Libro de Daniel (siglo ॥ a.C.) & Dn 6,1-28 & León reverente \\
\hline Anónimo (siglo Xv) & Corónica del Çid Ruy Díaz & León reverente \\
\hline Anónimo (siglo Xv) & Amadís de Gaula & Leona reverente \\
\hline
\end{tabular}


Posteriormente, el escritor romano en lengua griega Claudio Eliano (170249) relata en su Historia de los animales (libro VII, 48) una versión muy similar a la de Aulo Gelio acerca del león agradecido por la curación de Androcles y luego la protección brindada por el león no solo no devorándolo, sino también defendiéndolo de un leopardo que le arrojan al esclavo teniéndolo por brujo. En la versión de Eliano, Androcles cometió algún crimen por el que debió huir de su amo, mientras que en la de Gelio, escapó del maltrato injusto de su amo.

Tanto Gelio como Eliano ponen de modelo de conducta digna la de los animales ya que esta se adecua a los fines de su naturaleza, a diferencia de la de los humanos, que a menudo actúan en contra de los parámetros de la justicia. Ambos autores parten del principio teórico estoico que enseña que la Naturaleza o divindad universal dota a los animales de los medios suficientes para hacer un justo uso de sus miembros, con vistas a la defensa y conservación (Vara Donado 13).

En este mismo sentido, aparece en los exempla medievales el león, dotado de virtudes por ser de noble naturaleza y reflejar propiedades virtuosas impresas por Dios en todas sus criaturas.

Además del ya mencionado león agradecido, encontramos también a menudo unido a este el tópico del león reverente. En estos casos, la reverencia no surge de la gratitud, sino de su propia naturaleza (como rex ferarum), que le permite tanto reconocer la inocencia en el hombre como reverenciar a quienes poseen una alta nobleza moral. En esta línea aparece como antecedente más antiguo el Libro de Daniel, quien, condenado injustamente a ser devorado por los leones, resulta ileso y recibe la reverencia de una manada de leones hambrientos con los que lo encierran en un pozo. También existen numerosas leyendas de santos y mártires romanos, como la de Santa Tecla referida en la Leyenda dorada (Vorágine 908), a quien leones y leonas se niegan a devorar y, a quienes por el contrario reverencian, lo cual es interpretado en la tradición cristiana como un signo sobrenatural de la inocencia del mártir y de la protección divina proporcionada por una fiera, criatura ligada por naturaleza al Creador.

El flujo de obras que llegaban a América durante la etapa colonial era inmenso y, sobre todo, variado, como acertadamente afirma Irving: "Los embarques de novelas caballerescas hacia las Indias no comprendían, ni mucho menos, solo las de España; afluían también libros traducidos o adaptados del francés y de otras lenguas" (Irving 102). 


\section{"LA MALDONADA"}

En el famoso episodio de la Maldonada, Ruy Díaz (libro I, caps. 12-13) nos presenta una versión de este antiguo relato, aunque sometido a un proceso de transculturación: en el marco del sitio de los indígenas querandíes al asentamiento español en Buenos Aires en 1536, se nos narra la historia de una española, llamada la Maldonada, quien, no soportando más el hambre a causa del sitio de los aborígenes, abandona el campamento español y decide unirse a los indios para recibir alimentos. El hambre y la enfermedad llegaron a extremos tales que de aquí nacería también otro mito fundacional de la literatura argentina, el de la antropofagia entre españoles, relatado también por el bávaro Ulrico Schmidl y por Luis de Miranda. Veamos ahora cómo lo relata Ruy Díaz:

En este tiempo padecían en Buenos Aires cruel hambre, porque faltándoles totalmente la ración, comían sapos, culebras y las carnes podridas que hallaban en los campos: de tal manera, que los excrementos de los unos, comían los otros; viniendo a tanto extremo, que [...] comieron carne humana, así sucedió a esta miserable gente [...] Finalmente murió casi toda la gente, donde sucedió que una mujer española no pudiendo sobrellevar tan grande necesidad, fue constreñida a salirse del real e irse a los indios para poder sustentar la vida. (122)

Tras escapar del campamento español, la Maldonada, vagando por el monte, busca refugio en una cueva, que resultó estar habitada por una leona parturienta. La española reacciona en primera instancia con terror, mientras que la leona -que primero la percibe como una presa-, "usando de su real naturaleza" (123), teniendo "piedad" de ella, no la ataca. La mujer, a su vez, agradecida, decide ayudarla en el parto de dos leoncillos. La leona, que conseguía el sustento para sus crías, alimentaba también a la española. Mientras vivía allí, yendo a beber la mujer al río, es un día capturada por los indígenas y tomada por esposa por uno de ellos. Tiempo después, un capitán que sale a explorar la zona halla a la española conviviendo con una tribu y la entrega a Francisco Ruiz Galán, teniente de gobernador de Buenos Aires, quien manda castigarla, echándola a las fieras, para que la despedacen y coman como castigo por haber infringido las disposiciones de los conquistadores. Sin embargo, la fortuna hizo que llegara también la leona agradecida con sus leoncillos la cual, en lugar de devorarla, la protegió de otras fieras durante tres días. Así nos lo relata Guzmán: 
llevaron a la pobre mujer, la ataron muy bien a un árbol, y la dejaron como una legua fuera del pueblo, donde acudieron aquella noche a la presa gran número de fieras para devorarla, y entre ellas vino la leona a quien esta mujer había ayudado en su parto, y habiéndola conocido, la defendió de las demás que allí estaban, y que querían despedazarla.

Hasta que regresaron unos soldados y, encontrándola viva, admirados por la humanidad de aquella leona, la desataron y llevaron consigo. El animal mostró la humanidad que no tuvieron los hombres, su real ánimo y gratitud y, de esta manera, ganó la libertad la Maldonada, igual que Androcles.

Este relato presenta un esquema narrativo idéntico al del relato tradicional:

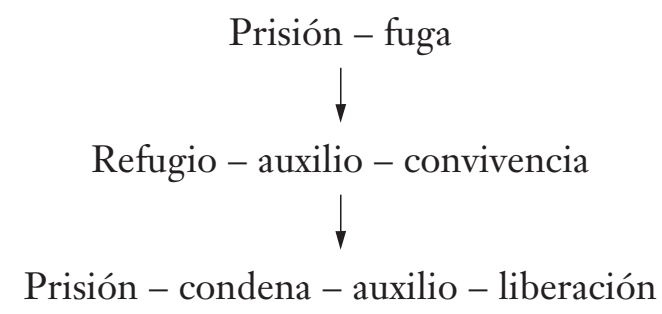

Sin embargo, no se debe perder de vista que la intención autoral de la obra de Guzmán es en principio extraliteraria, es decir, que el asunceno lo que pretende es "documentar" hechos históricos y no crear una obra literaria. Tanto insiste Guzmán en darle credibilidad a su relato que ciertos historiadores sostienen la realidad histórica de la Maldonada, así como de figuras como Lucía Miranda, de enorme peso literario, cuya historicidad ha sido puesta en duda por carecer de pruebas que acrediten su existencia histórica. Félix de Azara, por su parte -quien niega vehementemente la historicidad del relato de la Maldonada-, afirma la realidad histórica del relato de Lucía Miranda (Guérin 67). Otros historiadores, como Paul Groussac, por el contrario, lo descalifican como texto histórico debido a ciertas imprecisiones, sobre todo en las fechas de los acontecimientos que narra Guzmán.

Debido a las variadas opiniones en cuanto a la autenticidad y la credibilidad históricas de los relatos de Guzmán, puede establecerse una división en tres grupos de acuerdo con sus posturas: los historiadores y críticos que los aceptan como históricos y los reescribieron como tales, los que los aceptaron como históricos y los reescribieron como ficcionales y los que los consideran meramente literarios por ser sus referentes ficcionales (Guérin 68). 
Por su parte, para otorgarle más veracidad al relato, Ruy Díaz afirma: "Esta mujer yo conocí, y la llamaban la Maldonada" (129), destacando su calidad de testigo, no de los hechos narrados, pero sí de su existencia histórica.

Este es uno de los primeros desafíos que nos presenta Díaz de Guzmán, la hibridez de su obra histórico-literaria. Básicamente, como bien distingue Walter Mignolo (57), existen dos grupos de textos en el corpus colonial hispanoamericano: unos más historiográficos y otros de mayor carácter literario. A qué grupo pertenece con certeza nuestra obra sigue en plena discusión y preferimos abordarlo entonces como un texto narrativo plenamente híbrido.

En este punto resulta altamente pertinente mencionar la tesis del filósofo de la historia Hayden White, quien sostiene que, en realidad, todo historiador ficcionaliza la historia al introducir un elemento literario o de ficción en sus narraciones. Este elemento se justifica por la dependencia que sufre el discurso histórico de las técnicas del lenguaje figurativo, a la hora de describir la realidad y de buscar estrategias para construir los relatos (136). La tesis de White explica cómo se entrecruzan y borronean los límites entre historia y ficción y por qué el cronista recurre a su propio bagaje cultural para usarlo como pergamino donde escribirá hechos históricos. Este es propiamente el caso de Guzmán, quien, intentando escribir la historia de la conquista de la que sus propios antepasados fueron protagonistas, recurre al uso de este tópico literario del león, cargando la historia de elementos ficcionales y, a la vez, la literatura de elementos históricos. Incluso esta narración podría tratarse de una amplificación de cuentos de soldados, donde ya casi no se reconoce el hecho histórico que sirvió de núcleo (Caillet-Bois 111).

Vale mencionar que el hecho mismo de incluir un león en la región rioplatense representa un acto de transculturación, ya que sabemos que no existían tales fieras, sino otro tipo de felinos como el puma o el jaguareté.

Este elemento literario presente en la obra de Guzmán, surgido fuera del propio contexto cultural rioplatense, sufrirá un proceso de descontextualización y recontextualización, el cual dará lugar al fenómeno de la transculturación, siendo reimplantado en el nuevo lugar del hispanismo, adaptándose a las circunstancias históricas, en las que reina el enfrentamiento con la alteridad, representada por los pueblos originarios. La transculturación de este tópico a estas zonas le otorgará una nueva carga semántica y, sin perder la original función didáctica que buscaban los antiguos y los medievales, 
desempeñará otras funciones acordes al nuevo espacio cultural hispánico, cobrando un sentido más amplio, respondiendo a las necesidades estéticoculturales del nuevo espacio colonial, pasando a ser parte de la nueva cultura y sociedad nacientes, fungiendo como vehículo de exaltación de la epopeya española en tierras rioplatenses, como semilla de una visión dicotómica (civilización y barbarie) causante de la tensión y el enfrentamiento con las culturas locales, como marcador de pautas de comportamiento para los colonos, como crítica a los excesos de los conquistadores, como defensa de la situación de la mujer, como parámetro estético para la literatura fundacional rioplatense y como paradigma del padecimiento de los colonos (en vista a su exaltación).

Explicaremos a continuación estas funciones para clarificar su sentido. El autor exalta la conquista como una gesta heroica y loable y además escribe su obra "con propósito reivindicatorio para hablar de las hazañas de sus antepasados y de sus contemporáneos" (Caillet-Bois 105). No obstante, esto no le impide ejercer la crítica a los excesos de ciertos conquistadores. Asimismo, resulta verdaderamente asombrosa y destacable la defensa que hace de la mujer y la comprensión que manifiesta ante el hecho de que haya sacrificado su castidad en pos de la supervivencia. Ruy Díaz defiende fervientemente la inocencia de la Maldonada quien, si bien cometió una transgresión (desamparar a los suyos y unirse a los indios), lo hizo movida por el hambre. Por el contrario, acusa el cronista de "crueldad casi inaudita" (129) a quien condenó a la mujer, revelando incluso la posible identidad del perpetrador de la salvaje sentencia:

Por este suceso [no haber sido atacada por la fiera] se ve no haber merecido el castigo a que la expusieron, pues la necesidad había sido causa a que desamparase a los suyos y se metiese entre aquellos bárbaros. (129)

Esta actitud comprensiva por parte de Guzmán puede responder, según Guérin, a la de Carlos v y Felipe II, quienes, ante situaciones similares, optaron por perdonar la deserción y hasta el canibalismo de sus súbditos cuando estos tuvieron lugar motivados por el hambre extrema, como consta en la cédula real de 1536, reafirmada por Felipe II en 1570:

el Rey, informado de que hay "españoles" que se ausentaron del "Real" y se pasaron a los indios, y de que allí esos "cristianos" "viven" como los indios, ya que "han comido carne humana", y considerando que lo han hecho por "las necesidades y hambres", manda a su gobernador que "si 
algunos cristianos [...] se quisieren venir a esa provincia a estar e residir con los cristianos españoles que en ella residen, los dejéis y consintáis en venir a esa provincia y estar en ella y no procedáis contra ellos". (Guérin 74)

Nuestro autor inserta este relato maravilloso para llamar nuestra atención sobre diferentes facetas de la realidad histórica colonial: la hambruna durante el sitio y otros sufrimientos que conllevan la conquista de esas tierras, la difícil situación de la mujer, el enfrentamiento con la alteridad, y la polarización "bárbaros-civilizados". Además, funciona como un indicador del orden social al que se aspira, a saber: la condena social ante la posible unión entre hombre indígena y mujer española, así como el castigo a los infractores de las leyes de los españoles, ya sea a causa de excesos cometidos por parte de estos o por la ruptura de tabúes.

La inclusión del relato de la crueldad de los españoles que intentan castigar a la Maldonada y de la convivencia de esta con los indios -sin cargar las tintas en su barbarie- actúa como un agente que aminora la tensión y la concepción maniquea de la dicotomía "civilización-barbarie". Se expone claramente esta idea en la cita a continuación:

La hibridez implica tanto la expresión de categorías tabuizadas en el debate multicultural como el miedo y la alienación frente a lo extraño y el reclamo de patria e identidad, pero no en un sentido de exclusión, sino de negociación. (Toro 16)

En cuanto a la transformación del tópico a consecuencia de su migración a un lugar extraño, hemos visto que la circunstancia de la espina clavada en la pata de un león desaparece y es reemplazada por la de una leona parturienta. En este punto debemos preguntarnos (con vistas a responder a uno de nuestros postulados) si es esta circunstancia una invención del propio Ruy Díaz o si se basó en otra fuente española.

A la hora de abordar las relaciones intertextuales entre los textos hispanoamericanos y los europeos, topamos con un entramado difícil de desenmarañar, que no permite determinar fácilmente los vínculos de modo directo. La teoría de la intertextualidad postula en sí misma que no existe un vínculo, sino una cadena de relaciones entre textos de todos los tiempos; de modo que la prudencia se impone a la hora de determinar o excluir relaciones intertextuales. Por otra parte, en un texto americano del siglo XVII se agrega otra dificultad, a saber, la de no disponer a menudo de certezas para 
verificar con rigor científico si el autor americano tuvo o no contacto con determinada obra europea. Contamos, en la mayoría de los casos, con testimonios indirectos de los libros que llegaban a América y de las preferencias literarias del siglo XVI. Podemos mencionar como claras pruebas indirectas del enorme tráfico de libros hacia América las cartas de los reyes, exigiendo la prohibición de ciertas obras consideradas "dañinas para la formación moral de los indígenas" y exhortando al cumplimiento de anteriores decretos reales, nacidos de la preocupación por la avidez lectora de los españoles, criollos y mestizos en América.

Una de las cosas mas dañosas a la república cristiana es la elección de libros torpes y de caballerías, lo que no sirve de algún buen afecto sino de revivir las imaginaciones de torpes y lascivos deseos y de vanas y mentirosas fábulas, y principalmente se imprimen estas vanidades en gente moza con gran detrimento de sus almas, las cuales se corrompen con los dichos libros y se encienden en fuegos, y por ellos empiezan a aprender e intentar lo que no sabían ni habían oído por otras vías: por lo cual mandamos a todas las personas, hombres y mujeres, de todo nuestro obispado de cualquier estado o condición que sean, que so pena de excomunión mayor, dentro de cuatro días de publicación de esta constitución sinodal, nos traigan y envíen a las casas de nuestra morada todos los libros que se titulan Dianas, de cualquier autor que sean, y el libro que se titula de Celestina, y los libros de caballerías, y las poesías torpes y deshonestas. (Serrano Redonnnet 162)

Este decreto surge a raíz de la orden real de sacar de circulación estos libros por considerarlos dañinos. El rey Fernando, en 1506, ordenaba "para el buen gobierno de las Indias" que no se permitiera la venta de los libros profanos, frívolos o inmorales, a fin de que los indios no se aficionasen a leerlos (Irving 80). El 4 de abril de 1531 la reina, en un pliego de instrucciones que da a la Casa de Contratación de Sevilla, exige lo mismo, teniendo esta orden real fuerza de ley:

Yo he seydo ynformada que se pasan a las yndias muchos libros de Romance de ystorias vanas y de profanidad como son el amadis y otros desta calidad y por que este es mal exercicio para los indios e cosa en que no es bien que se ocupen ni lean, por ende yo vos mando que de aquí adelante no consyntays ni deis lugar a persona alguna pasar a las yndias libros ningunos de ystorias y cosas profanas salvo tocante a la Religion 
xriana e de virtud en que se exerciten y ocupen los indios e los otros pobladores de las dichas yndias por que a otra cosa no se ha de dar lugar. Fecha en Ocaña a quatro dyas del mes de abril de mil e quinientos y treinta y un años. Yo la Reyna. (Irving 80)

También contamos con inventarios en los autos de bienes de difuntos, donde aparecen, además de un enorme caudal de libros de caballerías (publicado entre 1558-1697), también muchos títulos de autores grecolatinos como Aulo Gelio, Esopo, Plinio, Plutarco, Séneca, Virgilio, entre otros (González Sánchez 112).

Asimismo, abundaban en Europa libros medievales con casos ejemplares de virtudes y debilidades humanas (exempla) muchos de los cuales, se hallaban en los autores antiguos. El Exemplum o "paradeigma", que se recoge como término técnico de la antigua retórica, a partir de Aristóteles, es utilizado también aquí como "historia breve, didáctica, que se inserta a manera de testimonio" (Curtius 98).

Como adelantamos, concretamente sospechamos una intertextualidad más directa con la obra de Sánchez de Vercial. No hemos encontrado un testimonio directo de la presencia de ejemplares del Libro de los Exenplos por A.B.C. de Sánchez de Vercial, sin embargo, intentaremos mostrar por qué nos parece altamente probable que se trate de la fuente más directa de Guzmán.

Por una parte, en su exemplum 186 Vercial nos narra -con leves diferencias- una historia con las características del episodio antiguo de Androcles y el león: la espina clavada en la pata, la curación, la comisión de un delito, la condena a muerte, la salvación gracias a la intervención del león agradecido y sus reverencias a su bienhechor. Ahora bien, ¿̇por qué nos inclinamos a pensar que esta es la fuente no solo de su acercamiento al relato antiguo, sino del origen del cambio de circunstancias en la crónica americana (o sea de la hembra en lugar del macho, del parto y la cría)? Probablemente se debe a que el exemplum inmediatamente anterior al recién mencionado, que también aborda el tema de la gratitud ejemplar de los animales, narra la historia de un hombre que, caminando por el desierto, encuentra las crías de una loba en apuros, encerradas en un hoyo. Este las rescata de allí y se las entrega a la loba-madre, la cual agradecida, le muestra un sendero secreto para que su benefactor siga su camino sin problemas. Sospechamos que Ruy Diaz combinó ambos exempla, o sea el 185 y el 186, escindiendo ciertos elementos, insertando y desechando otros, creando un contexto propio, dando lugar a un relato transculturizado. 
Fusión de lOS EXEMPLA DE SÁNCHEZ de VeRCIAL

\begin{tabular}{|c|c|c|}
\hline EXEMPLUM 185 & $\begin{array}{c}\text { EXEMPLUM } 186 \\
\text { (ANDROCLES Y EL LEÓN = AULO GELIO) }\end{array}$ & RELATO DE LA MALDONADA \\
\hline hombre protagonista & hombre protagonista & mujer protagonista \\
\hline loba parturienta en apuros & león & leona parturienta \\
\hline auxilio con los lobeznos & curación con espina clavada & auxilio con los leoncillos \\
\hline ------------- & comisión de un acto punible & comisión de un acto punible \\
\hline ------------- & condena a muerte & condena a muerte \\
\hline acto de gratitud & salvación de la muerte & salvación de la muerte \\
\hline ------------ & liberación del reo & liberación de la rea \\
\hline
\end{tabular}

Vemos en el esquema cómo el relato de Guzmán resulta una combinación de elementos de ambos exempla y de elementos propios del nuevo contexto que inserta el autor, generando una suerte de "exemplum americano". Es este un ejemplo de los procesos de hibridación y transculturalidad que menciona Alfonso de Toro:

En estos procesos de hibridación y transculturalidad, donde se recodifican tópicos, aplicando nuevos esquemas de pensamiento, dado el nuevo contexto cultural, importa poco el origen, y asumen el protagonismo el aspecto estético, su función social y su productividad. (Toro 17)

Como ejemplos concretos de esta afirmación, podemos mencionar el cambio en el género de la protagonista y del animal, que responde a diferentes motivos; sin embargo, nos interesa destacar que el autor pretende mostrar principalmente la inocencia de la española y, a su vez, la gravedad de la ruptura del tabú social acerca de la unión entre una mujer blanca y un indio, sentando así las bases de la sociedad colonial naciente. Por otra parte, en vistas a la verosimilitud del relato que Ruy Díaz presenta como completamente histórico, la tarea de comadrona resulta más apta para una mujer que quitarle una espina de la garra a una fiera, razón por la cual Guzmán se inclinaría por combinar ambos exempla.

Tanto en la tradición clásica como en la literatura medieval, aparece como hemos visto el león sumiso al hombre, movido por el agradecimiento por el auxilio recibido, tomándose al animal como paradigma de la gratitud, 
del que deberían aprender los humanos. Los exempla, que presentan acciones o conductas ejemplares, reflejan en el fondo los ideales de una sociedad.

Ahora bien, en nuestra introducción afirmamos que existe también la vertiente veterotestamentaria. Esta nos presenta al león reverente que, sin haber recibido ayuda alguna, reconoce la nobleza espiritual en el hombre, no solo no atacándolo, sino también reverenciándolo en una completa autosumisión. El más célebre modelo de este tópico es el Libro de Daniel, profeta condenado injustamente a morir en el pozo de los leones, ignorando sus verdugos que estos poseen la capacidad de reconocer la inocencia y la nobleza, de modo que no atacan a causa de la calidad espiritual del condenado.

En esta misma línea de origen veterotestamentario, se enmarca el episodio del Cid y el león: En la Corónica del Çid Ruy Díaz, tiene lugar el muy singular episodio: durante el descanso del héroe, se escapa un león de su jaula, ante el cual sus yernos huyen, mientras que sus caballeros lo rodean para protegerlo. Por su parte, Rodrigo despierta y, lejos de temerlo, recibe las reverencias del león que, avergonzado ante él, baja la cabeza pegando las fauces al suelo, dejándose guiar por el Campeador quien, con su diestra, lo toma por el cuello y lo lleva de vuelta a su jaula. Vale mencionar que la Corónica del Çid Ruy Díaz figura (junto con Amadís, Celestina y otros) entre los libros más impresos y difundidos en Sevilla, desde donde se traían a América (Irving 94).

Consideramos que, en el episodio de la Maldonada, Ruy Díaz hace una síntesis de ambas vertientes: el león actúa agradecido por el auxilio recibido (tradición grecolatina), y el narrador interpreta el hecho de que la Maldonada no sea atacada por el animal como un signo de la inocencia y nobleza de la española, tal como le sucede al Campeador (tradición veterotestamentaria). Eventos maravillosos que parecieran ser propiciados por fuerzas sobrenaturales que gobiernan las leyes de la naturaleza y las rompen de ser necesario para implementar la justicia que los hombres no pueden o no quieren ejercer.

\section{CONCLUSIONES}

La Argentina de Díaz de Guzmán representa un paradigma de hibridez cultural, donde concurren modelos literarios de espacios y tiempos remotos que se amalgaman con el substrato del Nuevo Mundo y confluyen finalmente en un crisol del que nacerán "espacios transculturales" (Toro 15). A este nuevo modelo transcultural pertenece el mismo Ruy Díaz de Guzmán, no solo como escritor y habitante de América, sino por ser él mismo paradigma del mestizaje, al ser descendiente de indios guaraníes y de hidalgos españoles. 
El primer autor mestizo del Río de la Plata, con sus imprecisiones y con sus aciertos históricos, realiza su aporte a la identidad de la cultura hispanoamericana, destacando la confluencia de culturas en América, no solo de la española, sino que, con el relato de "La Maldonada", hilvana otro eslabón en la larga cadena de transculturaciones que el imaginario europeo ha ido sufriendo a lo largo de los siglos, lo cual hemos querido ejemplificar a través del tópico del león reverente y agradecido.

Hemos constatado también que la recodificación de estos tópicos se realiza a través de la aplicación de nuevos esquemas de pensamiento. Esquemas que están ligados al nuevo contexto espaciotemporal y cultural, propiciando en la literatura rioplatense el surgimiento de nuevas categorías estéticas y nuevas funciones sociales, ausentes en instancias anteriores del periplo migratorio del tópico.

\section{Tradición veterotestamentaria (Libro de Daniel)}

[león reverente]

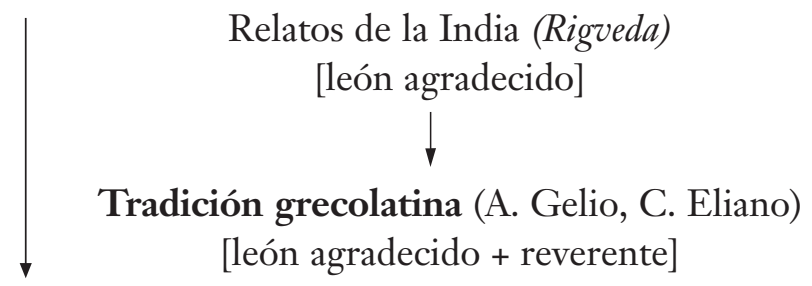

Tradición medieval y renacentista

(Sánchez de Vercial, Bestiarios, Libros de caballería,

La leyenda dorada)

[león reverente + león agradecido]

Migración y transculturación de Europa a América

$\downarrow$

Ruy Díaz de Guzmán, La Maldonada

Todo este análisis de fuentes, de migraciones y transformaciones del imaginario europeo a tierras americanas nos coloca frente a la historia del desarrollo de la identidad del Nuevo mundo, que se forja a través de estas migraciones, resemantizaciones y diálogo con la cultura autóctona, cuyo substrato lingüístico y cultural engendrará una cultura verdaderamente híbrida. 
La obra de Ruy Díaz merece ocupar un lugar relevante como obra fundacional de la literatura argentina ya que, con sus fines estéticos, retóricos, didácticos y testimoniales, nos dejó las huellas de los criterios bajo los que se fue gestando y desarrollando la cultura de buena parte de Sudamérica como nuevo lugar del hispanismo.

\section{OBRAS CITADAS}

Caillet-Bois, Julio. "La literatura colonial. Criollos y mestizos: Ruy Díaz de Guzmán”. Historia de la literatura argentina. Vol. 1. Dir. Rafael Arrieta. Buenos Aires: Peuser, 1958. 96-113.

Curtius, Ernst. Literatura europea y Edad Media latina. México: FCE, 1984.

Díaz de Guzmán, Ruy. La Argentina manuscrita. 1943. Ed. Enrique de Gandía. Madrid: Historia 16, 1986.

González Sánchez, Carlos. Los mundos del libro: medios de difusión de la cultura occidental en las Indias de los siglos XVI y XVII. Sevilla: Universidad de Sevilla, 1999.

Guérin, Miguel. "Discurso histórico y discurso ficcional en La Argentina, de Ruy Díaz de Guzmán”. Río de la Plata: culturas 11-12 (1991): 67-76.

Irving, Leonard. Los libros del conquistador. 1949. México: FCE, 2006.

Mignolo, Walter. "Cartas, crónicas y relaciones del descubrimiento y la conquista”. Historia de la literatura bispanoamericana. Madrid: Cátedra, 1982. $57-115$.

Sánchez de Vercial, Clemente. Libro de los exenplos por A.B.C. Ed. John Ke. Madrid: Ars Libris, 2000.

Serrano Redonnet, Antonio. "Prohibición de libros en el primer sínodo santiagueño (Tucumán)”. Revista de Filología Hispánica 5.2 (1943): 162-66.

Toro, Alfonso de. "Figuras de la hibridez: Ortiz: transculturación - Paz: hibridismo - Fernández Retamar: Calibán”. Alma cubana: transculturación, bibridez y mestizaje. The Cuban Spirit: Transculturation, Mestizaje, and Hybridism (Theorie und Kritik der Kultur und Literatur). Ed. Susanna Regazzoni. Frankfurt am Main: Vervuert, 2006. 15-36.

Vara Donado, José, ed. "Introducción”. Claudio Eliano. Historia de los animales. Madrid: Akal, 1989. 7-21.

Vorágine, Santiago de la. La leyenda dorada. Madrid: Alianza, 2011.

White, Hayden. Metahistoria: la imaginación histórica en la Europa del siglo XIX. 1973. México: FCE, 1993. 